\title{
Long-term outcomes of colorectal cancer patients with and without malignant large-bowel obstruction
}

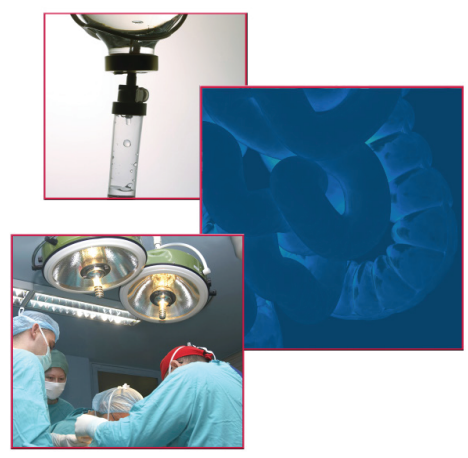

Shinya Munakata*,1, Yuta Murai ${ }^{1}$ Akihiro Koiuzumi ${ }^{1}$, Hisaki Kato1, Riku Yamamoto, Shuhei Ueda ${ }^{1}$, Satoshi Tokuda ${ }^{1}$, Shunsuke Sakuraba1, Tomoyuki Kushida ${ }^{1}$, Hajime Orita ${ }^{1}$, Mutsumi Sakurada ${ }^{1}$, Hiroshi Maekawa ${ }^{1}$ \& Koichi Sato ${ }^{1}$

1 Department of Surgery, Juntendo Shizuoka Hospital, Juntendo University School of Medicine, Shizuoka, Japan

*Author for correspondence: Tel.: +81 55948 3111; Fax: +81 55946 0514; smunaka@juntendo.ac.jp

\begin{abstract}
Aim: Colorectal cancer patients with malignant large-bowel obstruction (MLBO) require emergency treatment. Here we evaluated the long-term outcomes of recurrence and prognosis (not short-term mortality or nonocclusive cancer T1-T2) in colorectal cancer patients with and without an MLBO. Methods: We retrospectively analyzed the cases of the consecutive patients who underwent curative resection for colon cancer. We compared the groups regarding patients' age and gender, the tumor location, $\mathrm{T}$ and $\mathrm{N}$ factor, tumor stage, disease-free survival and overall survival. Results: The nonobstruction group had a significantly longer disease-free survival and overall survival compared with the MLBO group. Conclusion: MLBO was associated with cancer recurrence and poorer overall survival regardless of the staging and adjuvant chemotherapy.
\end{abstract}

First draft submitted: 4 January 2018; Accepted for publication: 24 April 2018; Published online: 25 July 2018

Keywords: adjuvant chemotherapy • colon cancer • disease-free survival • laparoscopy • malignant large-bowel obstruction • overall survival • staging • transanal decompression tube stoma • tumor location

Many patients with colon cancer exhibit locally advanced or metastatic disease at the time of presentation. An estimated $8-29 \%$ of colorectal cancer patients who present with an acute malignant large-bowel obstruction (MLBO) require emergency management, a stoma, self-expandable metallic stenting (SEMS) and a transanal decompression tube (TDT) [1]. These patients, who often are in poor physical condition due to several days of reduced intake, vomiting and weight loss, have operative risk leading to high mortality rates. The reported postoperative mortality following acute resection is $12-35 \%$ [2].

Initial colonic decompression using a minimally invasive procedure as a bridge to surgery might be an appropriate alternative to emergency operation. This approach creates time to optimize the patient's condition and prevents the stoma creation [3-5]. In addition, if a SEMS or TDT is not technically feasible, a decompressing stoma may be necessary to achieve a symptom-free condition for the patient. Although the mortality rate of these patients in the short term is high, little is known about the long-term outcomes of recurrence and prognosis [6,7]. In the present study, we analyzed the specific prognosis (except for short-term mortality such as progression of disease and postoperative complications) in colon cancer patients with and without an MLBO.

\section{Methods}

We reviewed cases of 256 consecutive patients who underwent curative resection for the treatment of their colorectal cancer at Juntendo University Shizuoka Hospital (Shizuoka, Japan) between January 2011 and December 2015. All cases of stage 0-I, IV, double cancers and rectal cancer were excluded from the analysis. Regarding stage 3 cancers, T1-2 cases were excluded because the tumor sizes were less than the colon obstruction group. The cases of the patients who died within 30 days postoperatively, classified as short-term mortalities, were also excluded from the analysis. All patients had histologically confirmed colon adenocarcinoma. Patients operated on by the authors

Future $\because$ Medicine 
Table 1. Baseline characteristics of the 148 patients who underwent surgical curative resection for colon cancer.

\begin{tabular}{|l|l|}
\hline Characteristic & $\mathrm{n}=148$ \\
\hline Age & 75 \\
\hline Sex (M/F) & $76 / 72$ \\
\hline T3/T4a/T4b & $133 / 9 / 6$ \\
\hline N0/N1/N2 & $88 / 45 / 15$ \\
\hline Stage II & 85 \\
\hline Stage III & 63 \\
\hline Location (Cecum/Ascending/Transverse/ Descending/Sigmoid) & $16 / 36 / 16 / 14 / 66$ \\
\hline Laparoscopic/open & $75 / 73$ \\
\hline Adjuvant chemotherapy & 80 \\
\hline
\end{tabular}

were given the option of open/laparoscopic surgery and the final decision depended on the surgeon's discretion. All patients were confirmed by pathology to have had a successful R0 resection.

We divided the patients into two groups: the MLBO group, with obstructive colon cancer $(\mathrm{n}=21)$ and the non-MLBO group, with nonobstructive colon cancer $(\mathrm{n}=127)$. The staging of all cancers is described according to the TNM Classification of Malignant Tumors, seventh edition (TNM 7th). We compared the following factors between the MLBO and non-MLBO groups: the patient's age, gender, the tumor location, $\mathrm{T}$ and $\mathrm{N}$ factor, tumor stage, the surgical approach (open vs laparoscopic-assisted colectomy), disease-free survival and overall survival. The surveillance protocol for the postoperative follow-up schedule was as follows: physical examination, serum carcinoembryonic antigen analysis, serum carbohydrate antigen 19-9 analysis, chest radiography and spiral abdominal computed tomography were performed every 6 months for 3 years and annually thereafter. Other procedures, such as colonoscopy and gastrofiberscope, were performed every 6-12 months depending on the status of the patient.

\section{Definitions}

The patients with a colon obstruction were identified based on clinical, radiological, endoscopic and intraoperative findings (complete obstruction) with oral perforation by tumor obstruction, which was confirmed using images, pathological findings and operative records. Cases of iatrogenic perforations and distant perforation sites were excluded.

Local recurrence was defined as recurrent tumors in the original tumor bed. Distant recurrence was defined as recurrent tumors outside the peritoneal cavity. Overall survival was defined as the duration from surgery to death or the last follow-up. Disease-free survival was defined as the duration from surgery to cancer recurrence. Colostomies were constructed on the transverse colon. After colostomy construction or TDT, patients received enteral feeding as soon as possible. Elective resection was performed within approximately 4 weeks.

\section{Statistical analyses}

The comparison of categorical variables was performed using the chi-square test or Fisher's exact test where appropriate. Continuous variables are presented as median values and were compared using the Mann-Whitney Utest. The length of time before the recurrence of colorectal cancer and death measured from the date of surgery were referenced against disease-free survival and overall survival time. Survival duration was calculated by the KaplanMeier method. The log-rank test was used for the comparison of the cumulative survival rate and disease-free survival in each patient group.

\section{Results}

\section{Patient characteristics}

Table 1 summarizes the clinicopathologic characteristics of the 148 patients who underwent surgical curative resection for colon cancer. The study group comprised 86 males and 72 females aged 35-92 years (median 75 years). Tumor location was the cecum (C) in 16 patients $(10.8 \%)$, followed by the ascending colon (A) in 36 patients $(24.3 \%)$, the transverse colon $(\mathrm{T})$ in 16 patients $(10.8 \%)$, the descending colon (D) in 14 patients $(9.4 \%)$ and the sigmoid colon in 66 patients (44.6\%). The laparoscopic approach was performed in 75 patients $(50.6 \%)$. Others were operated by the open approach. 


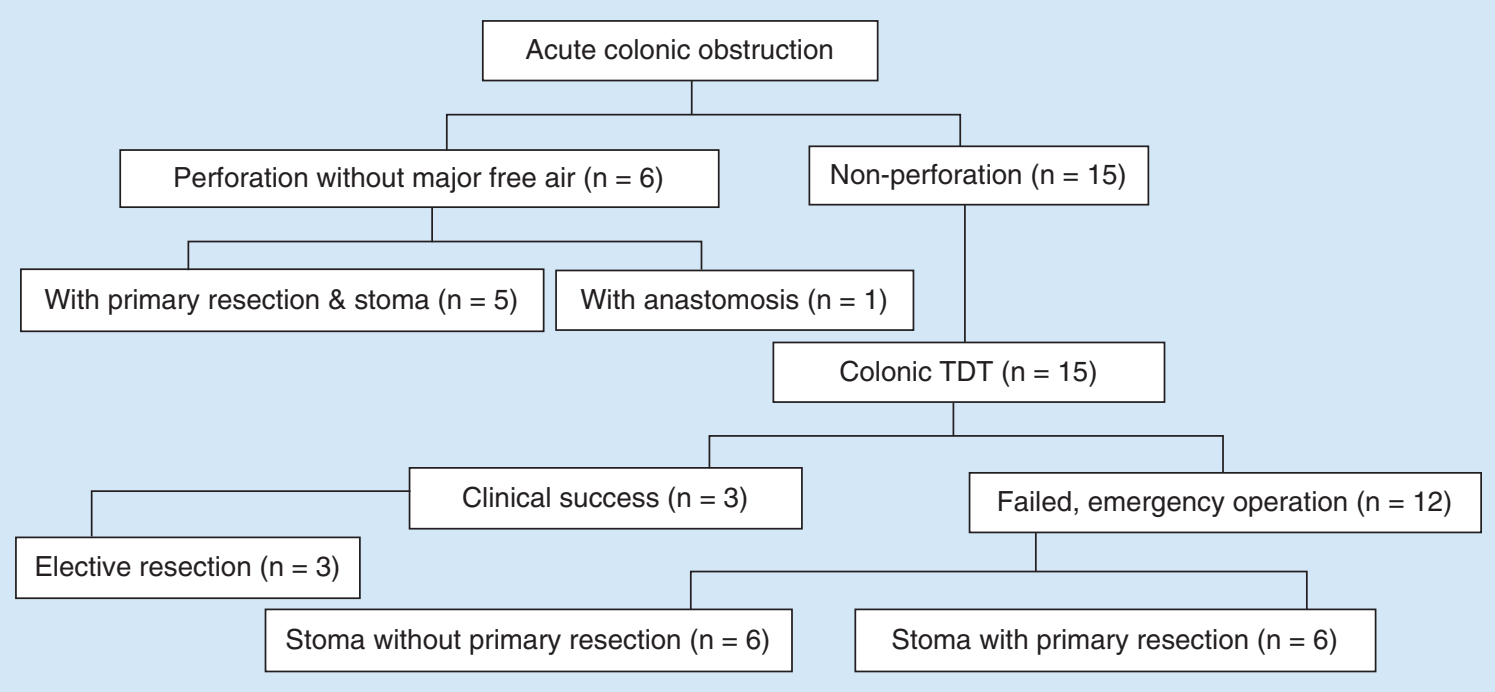

Figure 1. Flowchart of the management of patients with colon obstruction.

\begin{tabular}{|c|c|c|c|}
\hline Characteristic & MLBO $(n=21)$ & Non-MLBO $(n=127)$ & p-value \\
\hline Age & 72 & 76 & 0.46 \\
\hline $\mathrm{T} 3 / \mathrm{T} 4 \mathrm{a} / \mathrm{T} 4 \mathrm{~b}$ & $18 / 1 / 2$ & $115 / 8 / 4$ & 0.38 \\
\hline N0/N1/N2 & $14 / 6 / 1$ & $74 / 39 / 14$ & 0.62 \\
\hline Stage II & 14 & 71 & 0.35 \\
\hline $\begin{array}{l}\text { Location (Cecum/Ascending/ } \\
\text { Transverse/Descending/Sigmoid) }\end{array}$ & $0 / 0 / 2 / 7 / 12$ & $16 / 36 / 14 / 7 / 54$ & $<0.001$ \\
\hline Laparoscopic/open & $0 / 21$ & $75 / 52$ & $<0.001$ \\
\hline Adjuvant chemotherapy & 12 & 68 & 0.75 \\
\hline
\end{tabular}

MLBO: Malignant large-bowel obstruction.

The TNM-7th T categories were T3 in 133 patients (89.8\%), T4a in nine patients (6.0\%), T4b in six patients (4.1\%) and N0 in 88 patients (59.4\%), N1 in 45 patients (30.4\%), and N2 in 15 patients (10.1\%). Regarding TNM staging, 85 patients (57.4\%) were stage II and 63 patients (42.6\%) were stage III. The median follow-up duration was 38 months (range 2-76 months).

\section{The MLBO group's characteristics}

The flowchart of the colon obstruction management is depicted in Figure 1. Table 2 summarizes the details of the MLBO group. The patients in whom a temporary stoma creation was performed without primary resection $(n=6)$ proceeded to elective resection within approximately 4 weeks and stoma creation with primary resection $(n=6)$. The other patients $(n=3)$ decompressed by TDT proceed to elective resection. In the rest of the patients $(n=7)$, the perforation was caused, only to primary resection with stoma creation $(n=6)$ and with anastomosis $(n=1)$. Unfortunately, SEMS could not be performed due to health insurance circumstances in this periods.

\section{MLBO is revealed to be a prognostic factor}

In the comparison of the MLBO and non-MLBO groups, laparoscopic surgery was not performed in any patients in the MLBO group ( $\mathrm{p}<0.001)$. The tumor locations were partial between the two groups $(\mathrm{p}<0.001)$. No significant difference in adjuvant chemotherapy was noted between the groups (Table 3). As shown in Figure 2, the univariate $\log$-rank analysis showed that the non-MLBO group had a significantly longer disease-free survival $(\mathrm{p}<0.0001)$ 
Table 3. Characteristics of the patients with and without malignant large-bowel obstruction.

\begin{tabular}{|l|l|}
\hline Characteristic & $\mathbf{n = 2 1}$ \\
\hline Stoma creation without primary resection & 6 \\
\hline Stoma creation with primary resection & 6 \\
\hline Decompressed by transanal decompression tube proceed to elective resection & 3 \\
\hline Perforation with primary resection and stoma creation & 5 \\
\hline Perforation with anastomosis & 1 \\
\hline
\end{tabular}

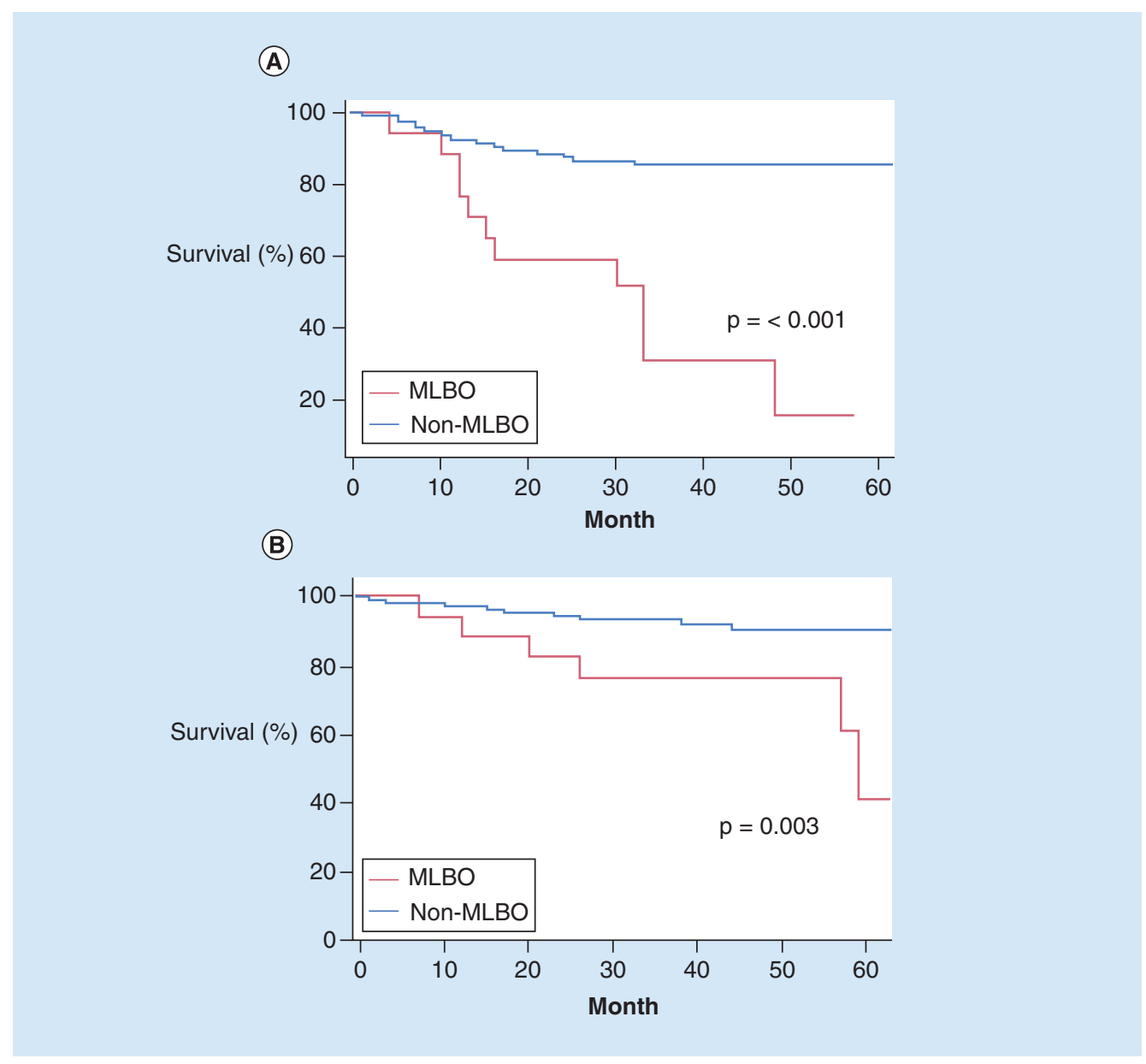

Figure 2. Survival curves of stage II-III colon cancer with or without malignant large-bowel obstruction. (A) Disease-free survival. (B) Overall survival.

MLBO: Malignant large-bowel obstruction.

and overall survival $(\mathrm{p}<0.003)$ compared with the MLBO group, indicating that MLBO was associated with the cancer recurrence and poorer overall survival regardless of the staging and adjuvant chemotherapy.

\section{Discussion}

The most common procedure for MLBO was traditionally emergency surgery without preoperative decompression. Preoperative decompression of colon distension has recently become the main treatment for MLBO. We had not performed SEMS in the present study period because Kim et al. showed that SEMS was associated with peritoneal seeding after perforation [8]. However, it was shown that the technical success rates were $100 \%$ for SEMS and $78.9 \%$ for TDT and significantly greater resolution of histopathological edema was achieved after the placement of SEMSs than after the placement of TDTs [9]. No significant difference in postoperative mortality was reported between SEMS and TDT [2]. Thus, although it is a controversial way to decompress the colon for obstructed 
colorectal cancer treatment, it is a possible option for decompression for an MLBO as a bridge to surgery. Future research is required to test the validity, reliability, reproducibility and clinical usefulness of SEMS.

Laparoscopy was considered to be difficult to perform for patients with colon obstruction within the limited working space, and there is a higher risk of causing inadvertent injury to the dilated bowel. In the present study period, we could not perform the laparoscopic surgery for patients with MLBO even if decompression succeeded. As recent studies showed that laparoscopic resection was completed in $74.2 \%$ patients in the SEMS group [10], this decompression therapy enables us to operate by the laparoscopic approach, but this should be confirmed by obtaining data regarding long-term outcomes for MLBO patients in randomized clinical trials the future.

Generally, there is a lack of evidence-based literature concerning laparoscopic surgery for locally advanced colon cancer. Indeed, cases of T3-T4 tumors, perforated tumors and acute bowel obstructions were excluded in large randomized, controlled trials comparing the laparoscopic approach with the open approach for colorectal cancer [11]. Here we observed that laparoscopic surgery did not compromise oncological outcomes in T3-T4 colon cancer patients compared with the open approach ( $\mathrm{p}=0.67$, data not shown). Therefore, at our institute, patients who will be treated with a SEMS benefit from a one-stage operation, without stoma creation and a safe primary anastomosis.

Despite the lack of robust data, the American Society of Clinical Oncology, the US National Comprehensive Cancer Network and the European Society for Medical Oncology guidelines recommend that adjuvant treatment be considered in patients with stage II colon cancer if specific high-risk features are present. These include perforation, T4 tumor, suboptimal lymph node sampling, poor differentiation, colonic obstruction, lymphovascular or perineural invasion, and positive resection margins. Kumor $e t$ al. indicated that adjuvant chemotherapy in patients with T4 disease only was associated with significantly improved recurrence-free survival, disease-specific survival, and overall survival. As for patients with MLBO, the advantage of adjuvant chemotherapy in overall survival only was observed in the overall high-risk stage II patients [12]. This may contribute to the worse short-term outcomes of MLBO patients. Therefore, our analysis of outcomes other than short-term mortality and nonocclusive cancer $\mathrm{T} 1-\mathrm{T} 2$ is necessary.

Petrelli et al. recently reported that right-sided cancers had a higher risk of mortality than left-sided cancers across all stages. The reasons for the inconsistent relationship between mortality and tumor location by stage are not clear. However, right-colon tumors are more likely to have V-raf murine sarcoma viral oncogene homolog B (BRAF) mutations, microsatellite instability (MSI), and the CpG island methylator phenotype [13]. On the other hand, as far as MSI is concerned, multiple studies have found that patients with MSI-positive tumors have a better overall prognosis, and it was reported that MSI status is an independent favorable predictor of survival [14]. Therefore, adjuvant chemotherapy is needed for patients with low-frequency MSI or microsatellite stable stage II or stage III colon cancer [14]. In Japan, MSI could not be measured due to health insurance requirements, but there were no significant differences between the right- and left-side colon cancers in the present study ( $\mathrm{p}=0.29$, data not shown).

This study is limited by its retrospective design and the small number of patients, which was due to our strict inclusion and exclusion criteria. More data are required regarding the prognoses of MLBO patients, and thus further studies are warranted.

\section{Summary points}

- Colorectal cancer patients with malignant large-bowel obstruction (MLBO) are frequently excluded from survival analyses.

- We retrospectively analyzed the cases of 148 consecutive patients who underwent curative resection for colon cancer.

- The 21 colon cancer patients with MLBO had worse disease-free survival and overall survival compared with the 127 non-MLBO patients, even when we excluded short-term mortality and nonocclusive T1-T2 cancer cases. 
Financial \& competing interests disclosure

The authors have no relevant affiliations or financial involvement with any organization or entity with a financial interest in or financial conflict with the subject matter or materials discussed in the manuscript. This includes employment, consultancies, honoraria, stock ownership or options, expert testimony, grants or patents received or pending, or royalties.

No writing assistance was utilized in the production of this manuscript.

\section{Ethical approval}

This study was performed in accordance with the ethical standards of the Committee on Human Experimentation of our institution (Institutional Review Board No. 539 29.336).

\section{Open access}

This work is licensed under the Attribution-NonCommercial-NoDerivatives 4.0 Unported License. To view a copy of this license, visit http://creativecommons.org/licenses/by-nc-nd/4.0/

\section{References}

Papers of special note have been highlighted as: $\bullet$ of interest; $\bullet \bullet$ of considerable interest

1. Deans GT, Krukowski ZH, Irwin ST. Malignant obstruction of the left colon. Br. J. Surg. 81, 1270-1276 (1994).

2. Tanis PJ, Paulino Pereira NR, van Hooft JE et al. Resection of obstructive left-sided colon cancer at a national level: a prospective analysis of short-term outcomes in 1,816 patients. Dig. Surg. 32, 317-324 (2015).

3. Sjo OH, Larsen S, Lunde OC, Nesbakken A. Short term outcome after emergency and elective surgery for colon cancer. Colorectal Dis. 11, 733-739 (2009).

4. Kobayashi H, Miyata H, Gotoh M et al. Risk model for right hemicolectomy based on 19,070 Japanese patients in the national clinical database. J. Gastroenterol. 49, 1047-1055 (2014).

5. Kawachi J, Kashiwagi H, Shimoyama R et al. Comparison of efficacies of the self-expandable metallic stent versus transanal drainage tube and emergency surgery for malignant left-sided colon obstruction. Asian J. Surg. pii: S1015-9584 (17) 30258-0 (2017)

6. Chen TM, Huang YT, Wang GC. Outcome of colon cancer initially presenting as colon perforation and obstruction. World J. Surg. Oncol. 15, 164 (2017).

-. Described the overall survival in colon cancer obstruction.

7. Amelung FJ, Consten ECJ, Siersema PD, Tanis PJ. A population-based analysis of three treatment modalities for malignant obstruction of the proximal colon: acute resection versus stent or stoma as a bridge to surgery. Ann. Surg. Oncol. 23, 3660-3668 (2016).

-• Described the outcomes of a bridge to the surgery approach using stent placement or stoma creation.

8. Kim SJ, Kim HW, Park SB et al. Colonic perforation either during or after stent insertion as a bridge to surgery for malignant colorectal obstruction increases the risk of peritoneal seeding. Surg. Endosc. 29, 3499-3506 (2015).

9. Takeyama H, Kitani K, Wakasa T et al. Self-expanding metallic stent improves histopathologic edema compared with transanal drainage tube for malignant colorectal obstruction. Dig. Endosc. doi: 10.1111/den.12585. [Epub ahead of print] (2015).

10. Ho KM, Chan KM, Kwok SY, Lau PY. Colonic self-expanding metal stent as a bridge to surgery in left-sided malignant colonic obstruction: an 8 year review. Surg. Endosc. 31, 2255-2262 (2017).

11. Chan DK, Tan KK. Laparoscopic surgery should be considered in T4 colon cancer. Int. J. Colorectal Dis. 32, 517-520 (2017).

12. Kumar A, Kennecke HF, Renouf DJ et al. Adjuvant chemotherapy use and outcomes of patients with high-risk versus low-risk stage II colon cancer. Cancer 121, 527-534 (2015).

13. Petrelli F, Tomasello G, Borgonovo $\mathrm{K}$ et al. Prognostic survival associated with left-sided vs right-sided colon cancer: a systematic review and meta-analysis. JAMA Oncol. 3, 2, 211-299 doi: 10.1001/jamaoncol.2016.4227. [Epub ahead of print] (2016).

14. Ribic CM, Sargent DJ, Moore MJ et al. Tumor microsatellite-instability status as a predictor of benefit from fluorouracil-based adjuvant chemotherapy for colon cancer. N. Engl. J. Med. 349, 247-257 (2003). 